
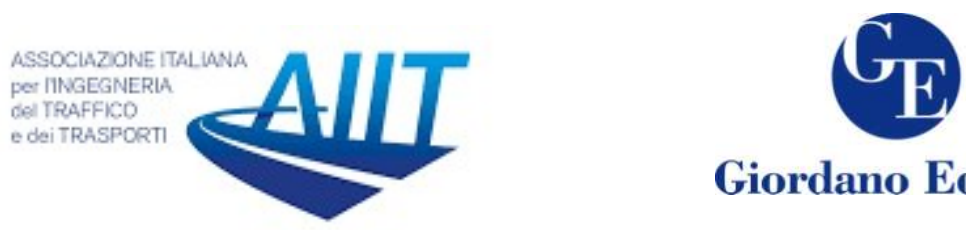

Giordano Editore

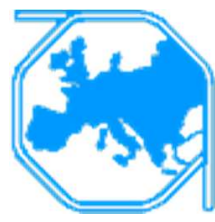

\title{
Results of Micro-Simulation Model for Exploring Drivers' Behavior on Acceleration Lanes
}

\author{
Giuseppe Cantisani $^{1 *}$, Giulia Del Serrone ${ }^{2}$, Giorgia Di Biagio ${ }^{3}$ \\ ${ }^{1}$ Associate Professor, Sapienza University of Rome, Via Eudossiana 18, 00184 Rome, Italy \\ ${ }^{2}$ PhD Student, Sapienza University of Rome, Via Eudossiana 18, 00184 Rome, Italy \\ ${ }^{3}$ Civil Engineer
}

\begin{abstract}
This study examines drivers' behavior on acceleration lanes, close to the convergence between the main and the secondary traffic streams, by means of traffic micro-simulations. Experimental data collected videotaping two acceleration lanes in Italy have been used to initially calibrate a simulation model and to validate it subsequently. The analyses had focused on both vehicles coming from the onramp, in terms of entrance points dispersion into the main traffic stream along the acceleration lanes, merging speeds, and acceleration rates reached, and on vehicles driving on the freeway right lane, in terms of vehicles categories, traffic volumes, and speeds. The maneuvers have been implemented in the TransModeler traffic simulation package and several scenarios have been considered, changing the traffic composition and the speeds at which drivers enter the acceleration lane from time to time. This led to obtain a large number of case studies, where the mutual influence between the two flows combined with the vehicle performances and the psychophysical characteristics of drivers, have led to an initial evaluation of the main variables in respect of which the required length for the specialized lanes depends. Road design guidelines' standards have been later compared to what was observed in reality and it can be claimed that the microscopic traffic model in some cases confirms the standards of road design guidelines while, in other cases, contradicts them.
\end{abstract}

Keywords: Micro-simulation; Acceleration Lanes; Road Design; Drivers’ behavior.

\section{Introduction}

The acceleration lanes are specialized lanes designed to allow the driver coming from an on-ramp to gradually increase his/her speed and merge into the target lane. The merging areas, in general, represent a part of a road where a secondary traffic flow, coming from a lane-changing maneuver, is introduced to the main traffic flow. In these circumstances, a sort of competition between the two flows arises, because each one of them wants to transit on the same space. Consequently, some turbulence could propagate and disturb both the flows in upstream and in downstream directions, up to causing relapses to the entire road system.

Therefore, as confirmed by various studies (Calvi \& De Blasiis, 2011 - Bokare \& Maurya, 2017), it is important to understand how drivers behave in these areas, in order

*Corresponding author: Giuseppe Cantisani (giuseppe.cantisani@uniroma1.it) 
to better managing the conflict points and improving the characteristics of the infrastructure especially in terms of acceleration lane length.

This study investigates the driver actual use of acceleration lanes by means of traffic micro-simulations in proximity to the convergence between the main and the secondary traffic streams. Thanks to the microsimulation model, calibrated and validated on reallife data, the geometrical and kinematic parameters to take heed of to improve the road design have been evaluated.

Secondly, it is very important to know the lengths that the acceleration lanes actually have, since the road designers have adopted over the years much more sacrificed solutions to realize these elements, than the ones provided by the theoretical models. In other studies, over the years, researchers have tried to understand which length of the acceleration lanes could be the more adequate to allow drivers to perform merging maneuvers safely (Sarhan et al., 2008 - Zhi et al., 2009 - Yang et al., 2016). In Italy, in particular, the discrepancy between theory and practice has been possible due to the fact that the current Italian design guidelines for the construction of road intersections (Ministerial Decree 19/04/2006) establishes only the general design principles concerning the infrastructure homogeneity. However, as to the methods of calculating the acceleration lane lengths, it refers only to some indications, leaving the possibility to the designer of evaluating the proper model to use, on a case-by-case basis, on its own responsibility.

In the light of the above, it has to be mentioned that in the technical literature several theoretical design models are presented, but with much uncertainties: they are based on experimental observations, which are significantly different from case to case. There is not a sufficiently solid theoretical background at the international level to propose different models from the existing ones, which consequently turn out to be far from the actual description of the phenomena.

To reach the gap between theory and practice, traffic microsimulation models, which can reproduce different driver behaviors depending on some parameters, have been used. Through these models, in fact, a tool for the determination of the correct length for the acceleration lanes has been defined, based on the variability of traffic volumes, travel speeds of freeway and ramp vehicles and driver-roadway-vehicle interactions, as a result of an accurate process of model calibration and validation.

The following section summarizes the literature related to existing design methods proposed by the Italian design standards (2006) and the AASHTO (2001), for the calculation of the length of the acceleration lanes.

Next, a description of the previous research work is presented, with all the calibration and validation phases of the microsimulation model that have led to understand how the guidelines remain out of touch with what really happens along acceleration lanes.

The last section summarizes the results of several different simulated scenarios, useful to define the required minimum acceleration lane length, capable to satisfy at least $85 \%$ of the merging maneuvers safely, without degradation of the level of service or a slowing down of traffic over the network. The idea is to utilize the microsimulation model to build up charts through which the exact length of the lane can be decided, combining some variables, like the on-ramp vehicle speeds at the beginning point of the acceleration lane, the traffic composition (\% heavy vehicles), the entering traffic volume from the on-ramp, and the traffic volume on the freeway right lane.

The paper concludes with a summary and recommendations.

\section{Reference standards}

The acceleration lane design is a key component of the road system in terms of safety: as has already been said, in fact, these lanes represent that part of the infrastructure where vehicles coming from an on-ramp try to merge into the target lane to travel on the same space of the main flow. It is therefore seen as essential to design the specialized lanes with an adequate length, to ensure that the drivers of the secondary 
flow can perform the lane changing maneuver safely, waiting for an acceptable gap to merge into, without the creation of queues.

International standards propose two distinct approaches to define the correct lane length of acceleration lanes, in relation to different driver pattern behaviors:

- The kinematic methods, proposed by various foreign technical standards (i.e. AASHTO Green Book)

- The functional and probabilistic analysis, as proposed, for example, by the Italian DM 19/04/2006

The main difference between the two approaches is that in the first one the acceleration lane consists of just two segments (Fig. 1): the acceleration section and the taper section. In the second one there is one more independent section, the waiting merging section (Fig. 2), which is designed based on the probabilistic distribution of the temporal distances between vehicles running on each lane.

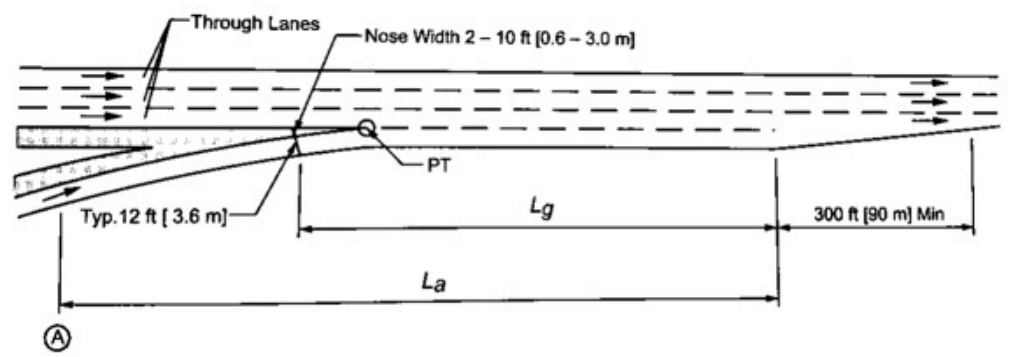

Figure 1: Typical Single-Lane Entrance Ramps - Parallel Design. La := acceleration length Lg:= gap acceptance length.

Source: A policy on Geometric Design of Highways and Streets-2018 7th Edition.

The AASHTO Green Book presents tabulated values of the minimum acceleration lane lengths required for the parallel-type entrances, for various combinations of design speeds of the freeway and initial speeds on the ramp. The desired distance increases with the increase of the merging speed and the ramp grade.

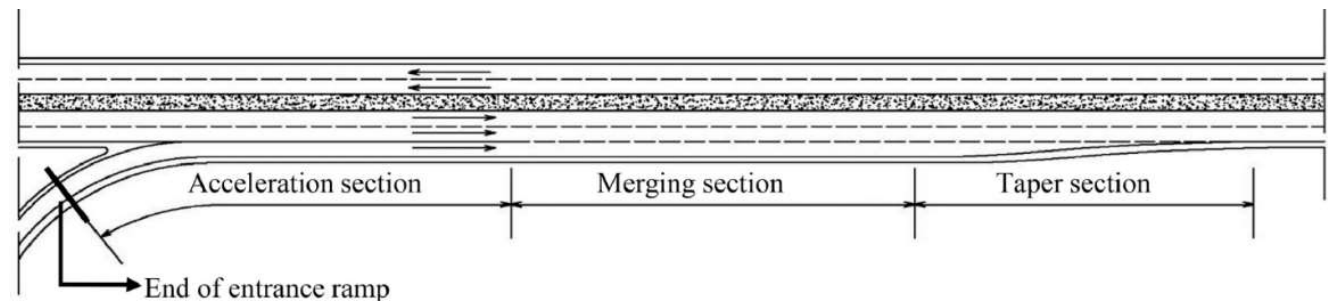

Figure 2: Entrance Ramp.

Source: Norme funzionali e geometriche per la costruzione delle intersezioni stradali Gazzetta Ufficiale-2006.

In the Italian guidelines, instead, the total length of an acceleration lane is defined by an additional section, dimensioned to allow drivers searching for acceptable gaps in the target lane to merge into the main traffic stream safely, once they have reached a speed consistent with the main traffic flow one. The length of this section can be determined according to different methods, based on traffic flows. The main ones are:

- Probabilistic methods

- Semi-empiric methods (i.e. Da Rios, 1985)

The first methods take into account the probability that a driver accepts a gap into the target lane, big enough to safely change lane. The distribution of the freeway traffic 
gaps, which depends on volumes and speeds, can be defined by an Erlang distribution, as shown also in a study by Kim \& Son (2003).

The semi-empirical methods propose to design the acceleration lane lengths adding to the acceleration section another section, but only under heavy flow conditions, which has to be sized as a function of the merging speeds.

\section{Initial results}

To understand the driver behaviors on acceleration lanes it was decided to use a microsimulation model, calibrated and validated by means of field observations along the E45 highway (European road), which is located in the Central area of Italy. In a previous work, indeed, (Cantisani et al., 2018) a traffic model was developed using the TransModeler microsimulation software (by Caliper corporation) and, following closely the FHWA proposed procedure, the next parameters had been calibrated (TransModeler User's Guide, 2008):

\subsection{Vehicle classification.}

As other studies show, i.e. Bokare and Maurya (2017), acceleration rates of the observed vehicles frequently differ from acceleration rates reported in the literature. For this reason, a piecewise constant acceleration model has been employed, classified according to vehicle categories and their Mass to Power Ratio, by some speed ranges, Tab.1.

Table 1: Maximum acceleration rates for vehicle classes and speed ranges.

\begin{tabular}{|c|c|c|c|c|c|c|c|c|c|c|}
\hline \multirow{2}{*}{$\begin{array}{l}\text { Percentage } \\
(\%)\end{array}$} & \multirow{2}{*}{ Name } & \multirow{2}{*}{ Description } & \multirow{2}{*}{ MPR } & \multirow{2}{*}{$\begin{array}{c}\text { Smax } \\
{[\mathrm{km} / \mathrm{h}]}\end{array}$} & \multicolumn{6}{|c|}{$\operatorname{amax}\left[\mathrm{m} / \mathrm{s}^{2}\right]$} \\
\hline & & & & & $\mathrm{s}<16$ & $16<s<32$ & $32<\mathrm{s}<48$ & $48<\mathrm{s}<64$ & $64<s<80$ & $\mathrm{~s}>80$ \\
\hline 50.00 & PC1 & $\begin{array}{c}\text { High } \\
\text { performance } \\
\text { passenger cars }\end{array}$ & 10 & 150 & 3.1 & 3.1 & 2.6 & 2.6 & 2.3 & 2.0 \\
\hline 31.00 & PC2 & $\begin{array}{c}\text { Middle } \\
\text { performance } \\
\text { passenger cars }\end{array}$ & 11 & 130 & 3.0 & 2.9 & 2.3 & 2.3 & 2.0 & 1.7 \\
\hline 4.00 & PC3 & $\begin{array}{c}\text { Low } \\
\text { performance } \\
\text { passenger cars }\end{array}$ & 12 & 100 & 2.0 & 1.8 & 1.3 & 1.1 & 1.0 & 0.9 \\
\hline 15.00 & PU & $\begin{array}{l}\text { Pickup trucks, } \\
\text { vans, and } \\
\text { SUVs }\end{array}$ & 28 & 100 & 1.1 & 1.3 & 1.6 & 1.9 & 1.0 & 1.0 \\
\hline
\end{tabular}

\subsection{Gap acceptance definition}

On freeways two different types of lane changing maneuvers can be distinguished: discretionary and mandatory. In the case of vehicles coming from an on-ramp, the decision to change lane to get into the target lane is an only choice. The driver has to find an acceptable gap into the main flow where to merge, before the end of the acceleration lane. Instead, a driver decides to change lane when he/she is traveling in the main flow for different reasons: probably he/she wants to accelerate to reach his/her desired speed.

So, as it is said in Balal et al. (2014), the parameters calibrated on real data to describe the non-linear gap acceptance model in TransModeler, were found to be different between the two types of lane changing (Tab. 2): 
Table 2: Calibrated parameters for the gap acceptance model.

\begin{tabular}{llcccc}
\hline & & \multicolumn{2}{c}{ Discretionary } & \multicolumn{2}{c}{ Mandatory } \\
\hline & & Lead & Lag & Lead & Lag \\
\hline Absolute minimum & Minimum [m] & 10.00 & 20.00 & 6.67 & 13.33 \\
Follower slower & $\beta_{1}^{g}[/ \mathrm{mps}]$ & 5.697 & 4.273 & 3.798 & 2.849 \\
Follower faster & $\beta_{2}^{g}[/ \mathrm{mps}]$ & 3.332 & 4.273 & 2.221 & 2.849 \\
Follower speed & $\beta_{3}^{g}[/ \mathrm{mps}]$ & 3.211 & 3.854 & 2.141 & 2.569 \\
Remaining distance & $\beta_{4}^{g}[/ \mathrm{mps}]$ & - & - & 0.10 & 0.10 \\
Sigma & $\varepsilon_{i}^{g}[\mathrm{~m}]$ & 1.00 & 2.00 & 0.67 & 1.33 \\
\hline & & & & &
\end{tabular}

These parameters must serve to solve the equation of the gap acceptance model, proposed in the software:

$$
G_{g}^{i}=\exp ^{\left(\beta^{g} X_{i}+\varepsilon_{i}^{g}\right)}
$$

\subsection{Critical distance distribution}

The last calibrated part of the traffic model had been the distribution of the entry points along the acceleration lanes: the more the driver approaches the taper section the more he/she feels the urgency to carry out the lane changing maneuver. Down the acceleration lane, the percentages of the entries in the main traffic flow follow a bell regression trend, which has been described by the following formula:

$$
\mathrm{y}=358 x^{2.8}(1.14-x)^{2.5}
$$

\section{Materials and methods}

In this section a great deal of attention is paid to calculate the length really necessary for the drivers coming from the on-ramp to enter into the main flow, by using the previously calibrated model on the following features (Tab. 3):

Table 3: Characteristics of the two road categories.

\begin{tabular}{ccc}
\hline & Highway & Ramp \\
\hline Speed Limit $[\mathrm{km} / \mathrm{h}]$ & 110 & 50 \\
Free Flow Speed, FFS $[\mathrm{km} / \mathrm{h}]$ & 120 & 60 \\
Flow, Q [veh/h/ln] & 400 & 50 \\
Number of lanes, N & 2 & 1 \\
Lane width, L [m] & 3.50 & 4.00 \\
\hline
\end{tabular}

Once the main traffic stream design speed (v1), the presentation speed at the end of the $\operatorname{ramp}(\mathrm{v} 2)$, the main and the secondary traffic flows (Q1 and Q2) are known, an abacus to obtain the acceleration lane length can be realized.

Using the formerly calibrated and validated model, in fact, a lot of different scenarios have been simulated to define the required acceleration lane length to perform the maneuver. 


\subsection{Scenarios and conditions analyzed}

Firstly, the main factors influencing the acceleration lane length have been identified. Secondly, these factors have been changed several times to understand how driver behaviors change, in response to some changes in the boundary conditions. The factors are as follows:

- Presentation speeds, s2, through which vehicles approach the acceleration lane, once they left the on-ramp. This speed has been varied in a $30 \mathrm{~km} / \mathrm{h}$ to $80 \mathrm{~km} / \mathrm{h}$ range, the first value can be considered as representative for loop ramps, the second one for directional ramps.

Within the upper and lower limits, a discretization of the presentation speed has been carried out, every $10 \mathrm{~km} / \mathrm{h}$, obtaining six different possible values to generalize, as far as possible, the obtained results.

- Secondary traffic flows Q2 coming from the on-ramp, expressed in veh/h, of which 4 cases have been analyzed, starting from a low flow of $50 \mathrm{veh} / \mathrm{h}$, moving to a lowermiddle flow of $100 \mathrm{veh} / \mathrm{h}$, an upper-middle flow of $200 \mathrm{veh} / \mathrm{h}$ and finally a high flow of $400 \mathrm{veh} / \mathrm{h}$. The choice has been made to analyze a large number of cases, compatible with real situations.

- Main traffic speeds, s1, of which just the free flow speed of $120 \mathrm{~km} / \mathrm{h}$ has been analyzed, as it represents the highway design speed.

- Main traffic flows, Q1, traveling on the right lane of the highway, expressed in $\mathrm{veh} / \mathrm{h}$. Just the case of a lower-middle flow of $400 \mathrm{veh} / \mathrm{h}$ per lane has been taken into account, but other cases could be considered, like an upper-middle flow of $800 \mathrm{veh} / \mathrm{h}$ per lane. The choice was based on the real data collected for the microsimulation model calibration, so that a comparison could be made between the results obtained.

- $\%$ of heavy vehicles, for both the main and the secondary traffic streams. It has been seen, in fact, that the presence of heavy vehicles affects considerably the driver behaviors, as it is said in Gattis et al. (2008).

In the microsimulation model, ten sensors have been placed along the acceleration lane (Fig. 3), with a length of $10 \%$ of the total length of the lane each, excluding the taper section.

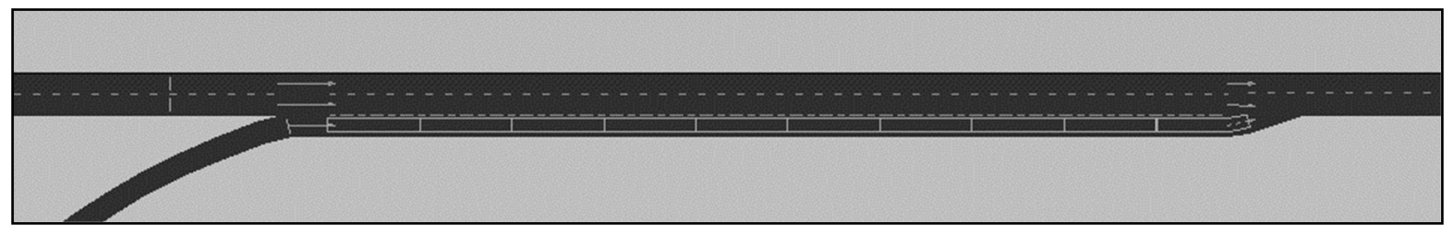

Figure 3: Sensors placement along the acceleration lane. Source: TransModeler

Different models have been built in TransModeler and several lengths have been assigned to the specialized lane, one for each model, from a minimum value of $80 \mathrm{~m}$ (excluding the taper section) to a maximum value of $350 \mathrm{~m}$. The intermediate lengths are: $100,130,170,220,280 \mathrm{~m}$.

Some parameters like entry speeds and entry points, in terms of numbers of vehicles entering the target lane from each sensor, have been extracted. Subsequently, the data have been summarized in tabular form, showing for each sensor the percentage of entry points, and then the percentage cumulative frequency of entry points along each lane 
section has been constructed. The summary of this analysis is presented below in Tab. 4 , where the results of a generic acceleration lane length have been averaged over 25 simulations, having the same input parameters:

Table 4: Percentage of entry points for each sensor, $\mathrm{s}_{0}=30 \mathrm{~km} / \mathrm{h}$.

\begin{tabular}{ccccccccccc}
\hline & $10 \% \mathrm{~L}$ & $20 \% \mathrm{~L}$ & $30 \% \mathrm{~L}$ & $40 \% \mathrm{~L}$ & $50 \% \mathrm{~L}$ & $60 \% \mathrm{~L}$ & $70 \% \mathrm{~L}$ & $80 \% \mathrm{~L}$ & $90 \% \mathrm{~L}$ & $100 \% \mathrm{~L}$ \\
\hline $\begin{array}{c}\% \text { of entry } \\
\text { points } \\
\%\end{array}$ & 0.00 & 4.80 & 0.40 & 14.00 & 14.40 & 15.20 & 19.20 & 10.40 & 9.60 & 12.00 \\
$\begin{array}{c}\% \\
\text { cumulative } \\
\text { frequency }\end{array}$ & 0.00 & 4.80 & 5.20 & 19.20 & 33.60 & 48.80 & 68.00 & 78.40 & 88.00 & 100.00 \\
\hline
\end{tabular}

Plotting the results in Fig. 4, cumulative frequency curves could be obtained, for different presentation speeds, once the main and the secondary traffic flows, $\mathrm{Q}_{1}$ and $\mathrm{Q}_{2}$, are established:

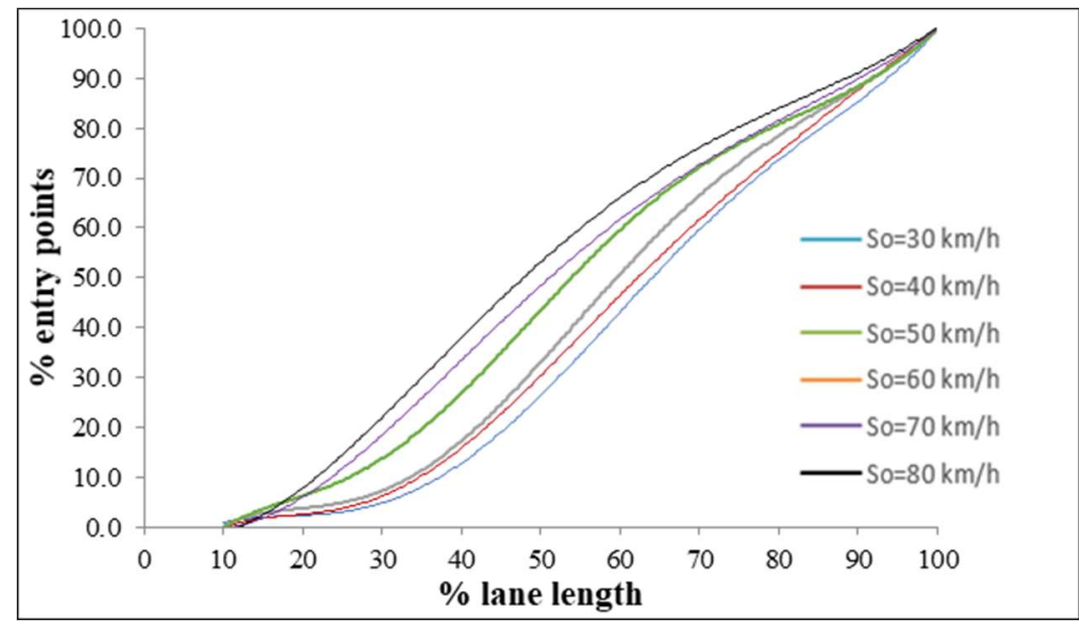

Figure 4: Cumulative frequency curves of entry points along the acceleration lane for different presentation speeds.

From Fig. 4 it can be concluded that there are no vehicles which merge into the main traffic flow in the first $10 \%$ of the lane length, then the number considerably increases in the central section of the lane, to have a slight decrease in the final part. For low entry speeds, the majority of vehicles wait for at least $40 \%$ of the lane length before they perform the lane changing maneuver because of the considerable speed difference between the two flows: such vehicles, in fact, need to accelerate to reach speeds close to the ones of the main flow. By increasing the presentation speed this behavior changes, until it becomes the opposite for a presentation speed equal to $80 \mathrm{~km} / \mathrm{h}$ : in the first half of the lane an increase in the number of vehicle lane changing maneuvers is seen and a decrease in the last part.

To define the correct length of the specialized lane, it was found at which percentage of the acceleration lane length the 90th percentile of the vehicles entering the acceleration lane occurs. If it happens at a greater distance than the $90 \%$ of the lane, an analysis with another traffic microsimulation model, built with a greater length, has to be carried out. Then, with a proportion, the appropriate lane length, for given flows $\mathrm{Q}_{1}$ and $\mathrm{Q}_{2}$ and a specific $\mathrm{s}_{0}$, can be determined, by requiring that the 90th percentile of the vehicles perform the lane changing maneuver in that distance. 
Once all the conditions for set values of $\mathrm{Q}_{1}$ and $\mathrm{Q}_{2}$ have been analyzed, six points on an abacus are obtained, one for each value of the presentation speed $\mathrm{s}_{0}$. These values can be plotted on the plane ( $\left.\mathrm{s}_{0}, \mathrm{~L}\right)$, as on the example shown below, Fig. 5:

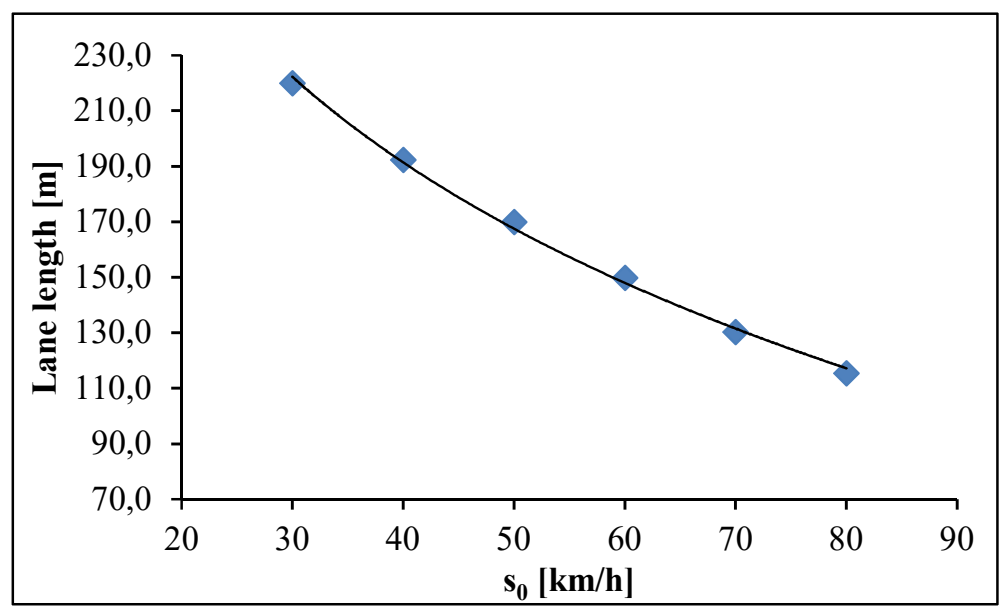

Figure 5: Trend line for the six values representative of the proper length of an acceleration lane, obtained with $\mathrm{Q}_{1}=400 \mathrm{veh} / \mathrm{h}$ and $\mathrm{Q}_{2}=400 \mathrm{veh} / \mathrm{h}$, depending on different $\mathrm{s}_{0}$ values.

The trend line has been defined through a logarithmic function, which best fits the location of the points.

\section{Results and conclusion}

The abacus obtained by the union of several curves at different values of Q2 can be presented in Fig. 6:

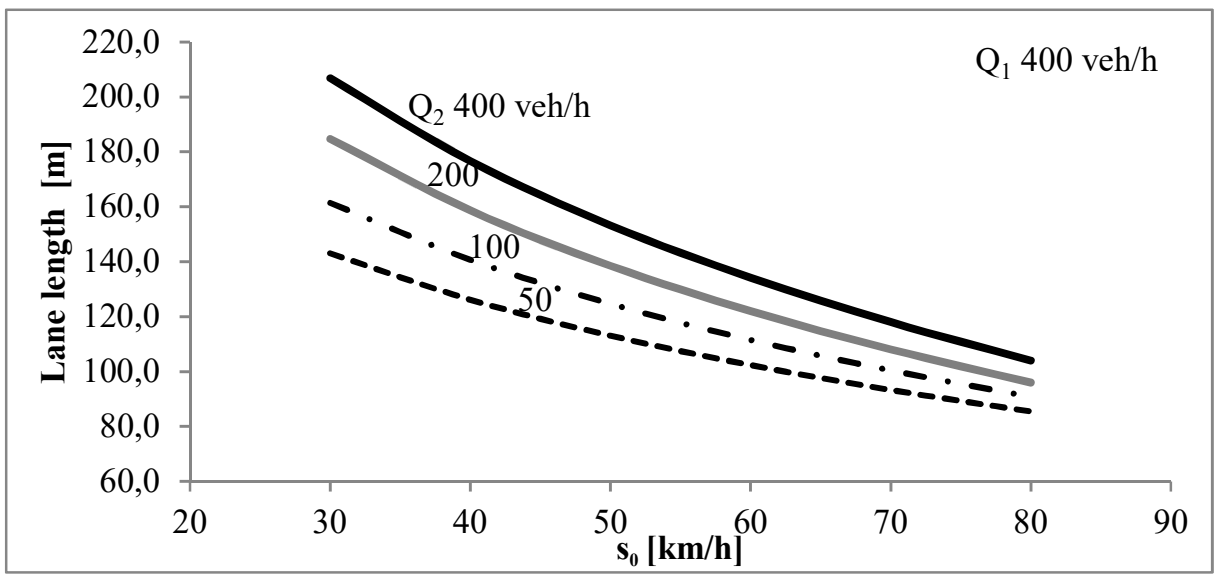

Figure 6: Length of an acceleration lane, obtained with $\mathrm{Q}_{1}=400 \mathrm{veh} / \mathrm{h}$ and $\mathrm{Q}_{2}=$ variable, depending on different $\mathrm{s}_{0}$ values.

Through this abacus, it is possible to calculate the required length of the acceleration lane. This length must guarantee that drivers can merge into the target lane, causing minimal disturbance for the main traffic flow.

To ensure this, the following situations should not happen in the area of flows overlapping:

- Formation of traffic jams 
- Waiting times

- Delays

- $\quad$ Poor or decreasing Level of Service (LOS)

Once the vehicle speed at the exit of the ramp, so, and the flow of the secondary traffic stream, $\mathrm{Q}_{2}$, are known, different abaci can be obtained varying the design speed and the main traffic flow, $\mathrm{s}_{1}$ and $\mathrm{Q}_{1}$. Another variable which can be changed is the vehicle fleet; the presented abacus refers to the mixed flow condition with $50 \%$ of PC1, $31 \%$ of PC2, $4 \%$ of PC 3 and $15 \%$ of ST. Every time a variable is changed, to obtain the points representative of the proper acceleration lane length, for each presentation speed, an average of 25 simulations has to be done.

The length information can be gained through the proposed graph, according to simple steps:

1. choose the graph characterized by the $\mathrm{Q}_{1}$ of interest (if not available, proceed by interpolation with other applicable graphs) and start to move into the graph from the presentation speed $\mathrm{s}_{0}$ at the end of the ramp;

2. move vertically from the $\mathrm{s}_{0}$ until the curve characterized by the $\mathrm{Q}_{2}$ representative of the case of interest is reached (if not directly available, proceed to interpolate curves also in this case);

3. move horizontally until the y-axis is reached, and find the length value representative of the proper one for the acceleration lane, under specific boundary conditions.

From these curves, it is clear that the higher the presentation speed the lower the length required to perform the maneuver for the vehicles, while for low presentation speeds the required length increases considerably. Furthermore, it is noted that the curves characterized by different flows $\mathrm{Q}_{2}$, pull away from each other as the presentation speed $\mathrm{s}_{0}$ decreases and the gap among the various curves is more pronounced. This result can be explained by the fact that the higher the number of vehicles coming from the ramp, the more reciprocal conditioning will be with the entry maneuvers. Focusing the attention on a single curve, at low presentation speeds a greater acceleration is needed to vehicles coming from the on-ramp to reach an adequate percentile of the speed of the current main one and execute the lane changing maneuver. Consequently, a greater length of the lane is required.

The obtained results, in the opinion of the authors, will be useful to produce, by means of the microsimulation model, various charts to use in the design analyses, in order to determine the length of the specialized lane, depending on the conditions (traffic, alignments, grade, ...) of each site.

\section{References}

AASHTO, M. (2001). Policy on geometric design of highways and streets. American Association of State Highway and Transportation Officials.

Balal, E., Cheu, R. L., Gyan-Sarkodie, T., \& Miramontes, J. (2014). Analysis of discretionary lane changing parameters on freeways. International Journal of Transportation Science and Technology, 3(3), 277-296.

Bokare, P. S., \& Maurya, A. K. (2017). Acceleration-deceleration behaviour of various vehicle types. Transportation research procedia, 25, 4733-4749.

Calvi, A., \& De Blasiis, M. R. (2011). Driver behavior on acceleration lanes: driving simulator study. Transportation research record, 2248(1), 96-103.

Cantisani, G., Del Serrone, G., \& Di Biagio, G. (2018). Calibration and validation of and results from a micro-simulation model to explore drivers' actual use of acceleration lanes. Simulation Modelling Practice and Theory, 89, 82-99.

Da Rios, G. (1999). Progetto di intersezioni stradali. 
Gattis, J. L., Bryant, M., \& Duncan, L. K. (2008). Acceleration lane design for higher truck volumes (No. MBTC FR 2094/3003). Mack-Blackwell National Rural Transportation Study Center (US).

Ministero delle Iinfrastrutture e dei Trasporti (2006). Norme funzionali e geometriche per la costruzione delle intersezioni stradali. Italian Ministry of Transportation Decreto Ministeriale: Rome, Italy.

Kim, S., \& Son, Y. (2003). Development of a new merge capacity model and the effects of ramp flow on the merge capacity. In Proceedings of the 82nd Annual Meeting of the Transportation Research Board (pp. 1-19).

Sarhan, M., Hassan, Y., \& Abd El Halim, A. O. (2008). Safety performance of freeway sections and relation to length of speed-change lanes. Canadian Journal of Civil Engineering, 35(5), 531-541.

TransModeler User's Guide (2008). Traffic Simulation Software. Caliper. MA, 18.

Yang, G., Tian, Z., Xu, H., \& Wang, Z. (2016). Recommendations for acceleration lane length for metered on-ramps. Transportation Research Record, 2588(1), 1-11.

ZHI, Y. F., ZHANG, J., \& SHI, Z. K. (2009). Research on design of expressway acceleration lane length and merging model of vehicle. China Journal of Highway and Transport, 22(2), 93-97. 